\title{
246. Some Characterizations of Certain von Neumann Algebras
}

\author{
By Marie CHODA and Hisashi CHodA \\ Department of Mathematics, Osaka Kyoiku University \\ (Comm. by Kinjirô KunUGi, M. J. A., Dec. 12, 1970)
}

In this note, we shall give some characterizations of finiteness and proper infiniteness of von Neumann algebras with respect to the density property of some subsets of von Neumann algebras.

1. Let $\mathfrak{S}$ be a Hilbert space and $\mathcal{L}(\mathfrak{S})$ the algebra of all (bounded linear) operators acting on $\mathfrak{S}$. By a von Neumann algebra $\mathcal{A}$ acting on $\mathfrak{S}$ we mean a weakly closed self-adjoint subalgebra (containing the identity $I$ ) of $\mathcal{L}(\mathfrak{S})$. A von Neumann algebra $\mathcal{A}$ is called finite if, for any $A \in \mathcal{A}, A^{*} A=I$ implies $A A^{*}=I$. This definition is equivalent to that, for any nonzero positive operator $A \in \mathcal{A}$, there exists a normal finite trace $\varphi$ on $\mathcal{A}$ such that $\varphi(A) \neq 0$.

B. Russo and H. A. Dye [9] showed that, in any von Neumann algebra $\mathcal{A}$, the convex hull of the set of unitary operators in $\mathcal{A}$ is weakly dense in the unit sphere of $\mathcal{A}$. Using the technique of Russo and Dye, we have the following:

Lemma 1. In any von Neumann algebra $\mathcal{A}$, the set of all unitary operators in $\mathcal{A}$ is strongly dense in the set of all isometric operators in $A$.

Proof. Let $V$ be an isometric operator in $\mathcal{A}$ such that $V V^{*}=P \neq I$ and $\mathfrak{M}$ be the range of $I-P$. Then $\left\{V^{n} \mathfrak{M} ; n \geqq 0\right\}$ are mutually orthogonal. Let

$$
\mathfrak{N}=\left(\bigoplus_{n=0}^{\infty} V^{n} \mathfrak{M}\right)^{\perp} .
$$

Then $\mathfrak{R}$ is invariant under $V$ and $V^{*}$. If $Q$ is the projection onto $\mathfrak{R}$, then $Q$ is dominated by $P$ and so that

$$
V Q V^{*}=V V^{*} Q=Q,
$$

or the restriction of $V$ to $\mathfrak{R}$ is unitary.

Given $\varepsilon>0$ and vectors $x_{1}, \cdots, x_{n} \in \mathfrak{F}$, choose $m$ such that

$$
\left\|\sum_{k>m} Q_{k} x_{i}\right\|<\varepsilon / 2
$$

for all $i$, where $Q_{k}$ is the projection onto $V^{k \mathfrak{M}}$. Let

$$
U=\left\{\begin{array}{l}
V \text { on } \mathfrak{R}+\mathfrak{M}+V \mathfrak{M}+\cdots+V^{m} \mathfrak{M} \\
\left(V^{m+1}\right)^{*} \text { on } V^{m+1} \mathfrak{M} \\
I \text { on } \underset{k>m+1}{\oplus} V^{k} \mathfrak{M} .
\end{array}\right.
$$

Then $U$ is a unitary operator in $\mathcal{A}$ such that 


$$
\left\|(U-V) x_{i}\right\| \leqq\left\|\sum_{k>m}(U-V) Q_{k} x_{i}\right\| \leqq 2\left\|\sum_{k>m} Q_{k} x_{i}\right\|<\varepsilon,
$$

for all $i$. Therefore the set of isometric operators of $\mathcal{A}$ is contained in the strong closure of the set of all unitaries of $\mathcal{A}$. Since the converse inclusion is obvious, this proves the theorem.

By the definition, any isometry in a finite von Neumann algebra is automatically unitary; hence Lemma 1 implies the following

Theorem 2. For a von Neumann algebra $\mathcal{A}$, $\mathcal{A}$ is finite if and only if the set of all unitary operators of $\mathcal{A}$ is strongly closed.

In addition, we have an another characterization of the finiteness of a von Neumann algebra with respect to the spectra of operators belonging to the algebra.

Let $\sigma(A)$ be the spectrum of an operator $A$ and $\alpha(A)$ the approximate point spectrum of $A: \lambda \in \alpha(A)$ if and only if there is a sequence $\left\{x_{n}\right\}$ of vectors of $\mathfrak{S}$ such that $\left\|(A-\lambda) x_{n}\right\| \rightarrow 0$.

In [3; Theorem 5], it is proved that a von Neumann algebra $A$ is finite if and only if the set of invertible operators of $\mathcal{A}$ is uniformly dense in $\mathcal{A}$. As a consequence, the following theorem follows:

Theorem 3. A von Neumann algebra $\mathcal{A}$ acting on $\mathfrak{S}$ is finite if and only if $\sigma(A)=\alpha(A)$ for any $A \in \mathcal{A}$.

Proof. For $A \in \mathcal{A}$, if $\lambda$ belongs to $\sigma(A)$, then $A-\lambda$ is not invertible. Since the set of invertible elements of $\mathcal{A}$ is uniformly dense in $\mathcal{A}, A-\lambda$ belongs to the boundary of the set of all invertible elements with respect to the uniform topology. Hence there is a sequence $\left\{A_{n}\right\}$ in $\mathcal{A}$ such that $\left\|A_{n}\right\|=1$ for any $n$ and $(A-\lambda) A_{n}$ converges uniformly to 0 (cf. [8; 1.5.4]). Therefore by [1; Ex. VII. 3.10], $\lambda$ belongs to $\alpha(A)$.

Conversely, assume that $\mathcal{A}$ is not finite, then there exists an isometric operator $V \in \mathcal{A}$ such that $V V^{*}<I$, that is, there exists a unit vector $x$ in the range of $I-V V^{*}$. Since $V^{*} x=0$, we have $0 \in \sigma\left(V^{*}\right)^{*}$ $=\sigma(V)$. Now, by the definition of the isometry, $0 \notin \alpha(V)$. This implies that $\mathcal{A}$ contains an operator $V$ such that $\sigma(V) \neq \alpha(V)$.

Remark. Even if an operator $A$ satisfies $\sigma(A)=\alpha(A), A$ needs not belong to a finite von Neumann algebra. In fact, if $A$ is hyponormal, then $A^{*}$ is cohyponormal and $\sigma\left(A^{*}\right)=\alpha\left(A^{*}\right)$ by [2], and a hyponormal operator in a finite von Neumann algebra is necessarily normal. Therefore, a non-normal cohyponormal operator $A$ satisfies $\sigma(A)=\alpha(A)$ but does not belong to any finite von Neumann algebra.

2. P. R. Halmos showed that, for an infinite dimensional Hilbert space $\mathfrak{S}_{\mathfrak{E}}$, the set of all commutators in $\mathcal{L}(\mathfrak{S})$ is strongly dense in $\mathcal{L}(\mathfrak{F})$ [7], and that the set of all unitary operators in $\mathcal{L}(\mathfrak{S})$ is weakly dense in the unit ball of $\mathcal{L}(\mathfrak{S})$ [6]. Using his technique in his proofs, we shall obtain a characterization and a necessary condition for the proper infiniteness of von Neumann algebras. 
A von Neumann algebra is properly infinite if it contains no nonzero finite central projection. An operator $A$ in a von Neumann algebra $\mathcal{A}$ is called a commutator in $\mathcal{A}$ if there exist operators $B$ and $C$ of $\mathcal{A}$ such that $A=B C-C B$.

The following theorem is a characterization of properly infinite von Neumann algebras in terms of the density of commutators:

Theorem 4. A von Neumann algebra $A$ is properly infinite if and only if the set of all commutators of $\mathcal{A}$ is $\sigma$-strongly dense in $\mathcal{A}$.

Proof. Let $\left(x_{i}\right)_{i=1}^{\infty}$ be a sequence of elements of $\mathfrak{S}$ such that $\sum_{i=1}^{\infty}\left\|x_{i}\right\|^{2}<+\infty$. Then, for any nonzero operator $A \in \mathcal{A}$, there exists a positive integer $N$ such that

$$
\sum_{n>N}\left\|x_{n}\right\|^{2}<1 / 20\|A\|^{2} .
$$

Suppose that $\mathcal{A}$ is properly infinite, then $\mathscr{A}$ contains a sequence $\left(E_{i}\right)_{i=1}^{\infty}$ of mutually orthogonal projections of $\mathcal{A}$ such that each $E_{i}$ is equivalent to $I$ and that $\sum_{i=1}^{\infty} E_{i}=I$. Therefore there exists a positive integer $M$ for which

$$
\sum_{n=1}^{N}\left\|\sum_{k=1}^{M} E_{k} x_{n}-x_{n}\right\|^{2}<1 / 20\|A\|^{2} .
$$

Put $P=E_{1}+\cdots+E_{M}$. Then $P \prec I-P$, so that there exists a partially isometric operator $V \in \mathcal{A}$ such that $V^{*} V=P$ and $V V^{*} \leqq I-P$. Setting $y_{k}=P x_{k}(k=1,2, \cdots, N), Q=A V^{*}$ and $B=Q V-V Q$, then

$$
B y_{k}=Q V y_{k}-V Q y_{k}=A y_{k} \quad(k=1,2, \cdots, N) \text {. }
$$

Since $\|B\| \leqq 2\|A\|$, we have

$$
\begin{aligned}
\sum_{n=1}^{N}\left\|A x_{n}-B x_{n}\right\|^{2} & \leqq \sum_{n=1}^{N}\left(\left\|A x_{n}-A y_{n}\right\|+\left\|A y_{n}-B y_{n}\right\|+\left\|B y_{n}-B x_{n}\right\|\right)^{2} \\
& \leqq 2 \sum_{n=1}^{N}\left(\left\|A x_{n}-A y_{n}\right\|^{2}+\left\|B y_{n}-B x_{n}\right\|^{2}\right) \\
& <2\left(\|A\|^{2}+\|B\|^{2}\right) / 20\|A\|^{2} \leqq 1 / 2
\end{aligned}
$$

On the other hand,

$$
\sum_{n=N+1}^{\infty}\left\|A x_{n}-B x_{n}\right\|^{2}<\|A-B\|^{2} / 20\|A\|^{2}<1 / 2
$$

Therefore, we have

$$
\sum_{n=1}^{\infty}\left\|A x_{n}-B x_{n}\right\|^{2}<1
$$

which proves that the set of the commutators of $\mathcal{A}$ is $\sigma$-strongly dense in $\mathcal{A}$.

Conversely, for a finite von Neumann algebra $\mathcal{A}$, the set of the commutators of $\mathcal{A}$ is not $\sigma$-strongly dense in $\mathcal{A}$.

But, if the set of the commutators of $\mathcal{A}$ is $\sigma$-strongly dense in $\mathcal{A}$, then it is also true in $\mathscr{A}_{E}$ where $E$ is a projection in the commutant $\mathscr{A}^{\prime}$ of $\mathcal{A}$, because the induction $A \rightarrow A_{E}$ is a normal homomorphism of $\mathcal{A}$ onto $\mathscr{A}_{E}$.

From this we can conclude that, if the set of the commutators of 
$\mathcal{A}$ is $\sigma$-strongly dense, then $\mathcal{A}$ does not have a finite part, that is, $\mathcal{A}$ is properly infinite.

The following theorem is a necessary condition for proper infiniteness of a von Neumann algebra:

Theorem 5. If $\mathcal{A}$ is a properly infinite von Neumann algebra, then the set of all unitary operators in $\mathcal{A}$ is weakly dense in the unit ball of $\mathcal{A}$.

Proof. By the assumption that $\mathcal{A}$ is properly infinite, $\mathcal{A}$ contains a sequence $\left(E_{i}\right)_{i=1}^{\infty}$ of mutually orthogonal projections such that $E_{i}$ is equivalent to $I$ and $\sum_{i=1}^{\infty} E_{i}=I$. Then $\mathcal{A}$ is spatially isomorphic to the tensor product $\mathcal{A}_{E_{1}} \otimes \mathcal{L}(\Re)$ where $\Re$ is an infinite dimensional separable Hilbert space $[4 ; \mathrm{p} .27]$. On the other hand, $\mathscr{A}_{E_{1}}$ is spatially isomorphic to $\mathcal{A}$ since $E_{1}$ is equivalent to $I$, so that $\mathcal{A}$ is spatially isomorphic to $\mathscr{A} \otimes \mathcal{L}(\Omega) . \quad$ From now on, we shall identify two von Neumann algebras $\mathcal{A}$ and $\mathscr{A} \otimes \mathcal{L}(\Omega)$.

Let $A=\left(A_{i j}\right)_{i, j=1,2, \ldots}\left(A_{i j} \in \mathcal{A}\right)$ be an operator in the unit ball of $\mathcal{A}$. Define $A_{n}=\left(A_{i j}\right)_{i, j=1,2, \cdots, n}$. Then $A_{n}$ is an operator in $\mathscr{A} \otimes \mathcal{L}\left(\Omega_{n}\right)$ with $\left\|A_{n}\right\| \leqq 1$, where $\AA_{n}$ is an $n$-dimensional subspace of $\AA$. Let $I_{n}$ be the identity operator in $\mathscr{A} \otimes \mathcal{L}\left(\Re_{n}\right)$, and let $S_{n}$ and $T_{n}$ be the positive square root of $I_{n}-A_{n} A_{n}^{*}$ and $I_{n}-A_{n}^{*} A_{n}$ respectively, then

is unitary. Furthermore, put

$$
V_{n}=\left(\begin{array}{cc}
A_{n} & S_{n} \\
T_{n} & -A_{n}^{*}
\end{array}\right)
$$

$$
U_{n}=\left(\begin{array}{ll}
V_{n} & 0 \\
0 & I
\end{array}\right)
$$

then clearly $U_{n}$ is unitary too.

Finally, let us define $x_{j}=\left(\delta_{i j} x\right)_{i=1,2, \ldots}$, where $\delta_{i j}$ is Kronecker's delta, then for any $x$ and $y$ in $\mathscr{S}$ we have

$$
\left(A x_{i} \mid y_{j}\right)=\left(U_{n} x_{i} \mid y_{j}\right) \quad \text { for } i, j=1,2, \cdots, n \text {. }
$$

This proves that the set of all unitary operators is dense in the unit ball with respect to the weak topology.

Theorem 4 has counterpart for non-atomic von Neumann algebras which is established by H. A. Dye [5].

Remark. In the proof of Theorem 4 , if $A$ is positive and $\|A\| \leqq 1$, then $U_{n}$ represents a projection of $\mathcal{A}$ if we replace $S_{n}$ with the positive square root of $A_{n}\left(I-A_{n}\right)$ and $V_{n}$ with

$$
\left(\begin{array}{cc}
A_{n} & S_{n} \\
S_{n} & I-A_{n}
\end{array}\right)
$$

Therefore, we have the following proposition: If $\mathcal{A}$ is a properly infinite von Neumann algebra, then the weak closure of the set of all projections of $\mathcal{A}$ fills out the positive part of the unit ball. 


\section{References}

[1] S. K. Berberian: Introduction to Hilbert Space. Oxford Univ. Press, New York (1961).

[ 2 ] _- : A note on hyponormal operators. Pacif. J. Math., 12, 1171-1175 (1962).

[3] H. Choda: An extremal property of the polar decomposition in von Neumann algebra. Proc. Japan Acad., 46, 341-344 (1970).

[ 4 ] J. Dixmier: Les algèbres d'opérateurs dans l'espace Hilbertien. GauthierVillars, Paris (1957).

[5] H. A. Dye: The unitary structure in finite rings of operators. Duke Math. J., 20, 55-69 (1953).

[6] P. R. Halmos: Normal dilations and extensions of operators. Summa Brasil., 2, 125-134 (1950).

[ 7 ] - : Commutators of operators. II. Amer. J. Math., 76, 191-198 (1954).

[ 8 ] C. E. Rickart: General Theory of Banach Algebras. Van Nostrand, Princeton (1960).

[9] B. Russo and H. A. Dye: A note on unitary operators in $C^{*}$-algebras. Duke Math. J., 33, 413-416 (1966). 